(http://www.cdc.gov/nchs/data/nhanes/nhanes_03_04/ nhanes_analytic_guidelines_dec_2005.pdf). (Accessed August 2, 2013).

Sharon Saydah, Giuseppina Imperatore, and Linda Geiss (e-mail: ssaydah@cdc.gov)
Division of Diabetes Translation, Centers for Disease Control and Prevention, Atlanta, GA 30341

(C) The Author 2013. Published by Oxford University Press on behalf of the Johns Hopkins Bloomberg School of

Public Health. All rights reserved. For permissions, please e-mail: journals.permissions @oup.com.

\title{
RE: "PREVALENCE OF DIAGNOSED AND UNDIAGNOSED TYPE 2 DIABETES MELLITUS AMONG US ADOLESCENTS: RESULTS FROM THE CONTINUOUS NHANES, 1999-2010”
}

The report by Demmer et al. (1) estimated the prevalence of diagnosed and undiagnosed diabetes in US adolescents. As investigators for the SEARCH for Diabetes in Youth Study (herein, the SEARCH Study), a US population-based registry of diabetes in youth (2), we acknowledge that providing valid estimates of the burden of diabetes in children, overall and by type, is an important and complex endeavor, but we wish to raise several concerns about the validity and representativeness of these findings.

The authors reported a prevalence of self-reported type 1 diabetes mellitus (T1DM) that is approximately $65 \%$ higher than that reported by the SEARCH Study in 2001 (3.8/1,000 vs. $2.3 / 1,000$, respectively) (3), which may be explained by temporal trends in T1DM prevalence, but also by unvalidated overreporting, both of which the authors mentioned. Surprisingly, the prevalence of self-reported type 2 diabetes mellitus (T2DM) (1.8/1,000 among those aged 12-19 years) was 4.3 times higher than previously reported by the SEARCH Study $(0.42 / 1,000$ for those aged $10-19$ years) and much closer to SEARCH Study estimates for American Indian youth (1.7/ 1,000 ), the racial group with the highest prevalence of T2DM.

We believe that the small number of adolescents screened, the imprecise case definition, and misclassification of diabetes type may underlie this. The authors defined T2DM on the basis of self-reported diet therapy or use of oral hypoglycemic agents with or without insulin. We agree that true diabetes cases who do not take insulin are likely to have T2DM; however, up to $11 \%$ of 8,879 youth with T1DM in the SEARCH Study are taking oral hypoglycemic agents in addition to insulin (4-6). Because cases of T1DM greatly outnumber those of T2DM, even low rates of misclassification of T1DM as T2DM will result in an overestimate of T2DM prevalence. Because insulin use varies among minority groups (4-6), differential misclassification by racial/ethnic group will likely occur.

Even higher estimates were reported in the fasting subsample, all of which were considered to have undiagnosed T2DM. The authors acknowledged that fasting glucose levels are variable in youth, and that a 1-time elevated value would not result in a diagnosis of diabetes; however, the impact of such problems is less well recognized. In the HEALTHY Study (7), only 1 of the original 6 youth with elevated fasting glucose was confirmed for a true prevalence of $1 / 6,358=$ $0.02 \%$. This compares to an unweighted prevalence of elevated fasting glucose of $0.17 \%$ in the National Health and Nutrition Examination Survey (NHANES) (1). In the Princeton Study of 2,501 elementary students (8), 7 youth with "near diabetes" were identified $(0.28 \%)$, but only 1 was confirmed to have T2DM, for a prevalence of $0.04 \%$. Thus, the prevalence of previously known $(n=2)$ and undiagnosed $(n=1) \mathrm{T} 2 \mathrm{DM}$ was $0.12 \%$; the weighted prevalence reported by Demmer et al. (1) was 3 times higher $(0.36 \%)$.

The authors make the case that the NHANES population is representative; however, their sample contains no American Indians or Asian/Pacific Islanders, 2 groups with the highest risk of T2DM, so it is unclear how representative the sample is for US youth at risk for diabetes. The explanation provided by the authors for the lower T2DM prevalence in the SEARCH Study (3) versus NHANES is that the SEARCH Study is not a geographically representative sample. The geographical areas included in the SEARCH Study were not based on random sampling; however, the SEARCH Study population is quite representative of the racial/ethnic, sex, education, and income distributions in the United States (9). Importantly, the SEARCH Study prevalence estimates are based on more than 6,000 cases with diabetes identified from a denominator of approximately 3.5 million youth compared with only 58 cases in fewer than 12,000 youth from NHANES.

The authors conclude that "T2DM accounts for approximately half of adolescent diabetes in the United States ..." $(1$, p.1). The SEARCH Study recently estimated that 18,919 youth aged 10-19 years in the United States had diagnosed T2DM, and 132,315 youth less than 20 years of age had T1DM, and that T2DM accounts for $12.5 \%$ of all diabetes cases in youth (9), which is substantially less than estimated by Demmer et al. (1). Given the small number of cases and the numerous problems with case definitions discussed above, we believe that the estimates provided by Demmer et al. based on NHANES data do not accurately reflect the prevalence of diabetes, especially T2DM, in US adolescents.

\section{ACKNOWLEDGMENTS}

Conflict of interest: none declared. 


\section{REFERENCES}

1. Demmer RT, Zuk AM, Rosenbaum M, et al. Prevalence of diagnosed and undiagnosed type 2 diabetes mellitus among US adolescents: results from the continuous NHANES, 1999-2010. Am J Epidemiol. 2013;178(7):1106-1113.

2. SEARCH Study Group. SEARCH for Diabetes in Youth: a multicenter study of the prevalence, incidence and classification of diabetes mellitus in youth. Control Clin Trials. 2004;25(5): 458-471.

3. The SEARCH for Diabetes in Youth Study Group, Liese AD, D'Agostino RB Jr, et al. The burden of diabetes among US youth: prevalence estimates from the SEARCH for Diabetes in Youth Study. Pediatrics. 2006;118(4):1510-1518.

4. Bell RA, Mayer-Davis EJ, Beyer JW, et al. Diabetes in non-Hispanic white youth: prevalence, incidence, and clinical characteristics: the SEARCH for Diabetes in Youth Study. Diabetes Care. 2009;32(suppl 2):S102-S111.

5. Lawrence JM, Mayer-Davis EJ, Reynolds K, et al. Diabetes in Hispanic American youth: prevalence, incidence, demographics, and clinical characteristics: the SEARCH for Diabetes in Youth Study. Diabetes Care. 2009;32(suppl 2):S123-S132.

6. Mayer-Davis EJ, Beyer J, Bell RA, et al. Diabetes in African American youth: prevalence, incidence, and clinical characteristics: the SEARCH for Diabetes in Youth Study. Diabetes Care. 2009;32(suppl 2):S112-S122.

7. HEALTHY Study Group, Kaufman FR, Hirst K, et al. Risk factors for type 2 diabetes in a sixth-grade multiracial cohort: the HEALTHY Study. Diabetes Care. 2009;32(5):953-955.

8. Dolan LM, Bean J, D'Alessio D, et al. Frequency of abnormal carbohydrate metabolism and diabetes in a population-based screening of adolescents. J Pediatr. 2005;146(6):751-758.

9. Pettit DJ, Talton J, Dabelea D, et al. Prevalence of diabetes mellitus in U.S. youth in 2009: the Search for Diabetes in Youth Study [published online ahead of print September 16, 2013]. Diabetes Care. (doi:10.2337/dc13-1838).

Dana Dabelea ${ }^{1}$, Jean M. Lawrence ${ }^{2}$, Catherine Pihoker $^{3}$, Lawrence Dolan ${ }^{4}$, Ralph B. D'Agostino, $\mathrm{Jr}^{5}$, Santica Marcovina ${ }^{6}$, and Elizabeth J. Mayer-Davis ${ }^{7}$, for the SEARCH for Diabetes in Youth Study (e-mail: dana.dabelea@ucdenver.edu)

${ }^{1}$ Department of Epidemiology, Colorado School of Public Health, Aurora, CO 80045

${ }^{2}$ Department of Research and Evaluation, Kaiser Permanente Southern California, Pasadena, CA 91101

${ }^{3}$ Department of Pediatrics, University of Washington, Seattle, WA 98105

${ }^{4}$ Department of Endocrinology, Children's Hospital

Medical Center, Cincinnati, OH 45229

${ }^{5}$ Department of Biostatistical Sciences, Wake Forest School of Medicine, Winston-Salem, NC 27157

${ }^{6}$ Department of Medicine, University of Washington, Seattle, WA 98105

${ }^{7}$ Department of Nutrition and Department of Medicine, University of North Carolina at Chapel Hill, Chapel Hill, NC 27599

DOI: 10.1093/aje/kwt277; Advance Access publication: November 15, 2013

\section{THE AUTHORS REPLY}

We appreciate the interest expressed by Saydah et al. (1) and Dabelea et al. (2) in regard to our recent article (3) addressing adolescent diabetes prevalence in the United States. The letters reinforce 2 limitations with which we fully agree, as demonstrated by our careful discussion of these points in our original article. Although our colleagues do not raise new perspectives but rather reinforce known limitations, their comments provide a valuable opportunity to further discuss the complexity of diabetes surveillance in youth.

Sayda et al. (1) comment on the low precision of prevalence estimates from sex and racial/ethnic subgroups, which stems from the very low prevalence of adolescent diabetes in general. We agree with this point, and to minimize the potential for overinterpretation of subgroup estimates, we did not include them in the abstract, avoided drawing conclusions about sex and racial/ethnic differences anywhere in the manuscript, and dedicated a full paragraph in the discussion to emphasize caution in interpreting the results. Importantly, although relative standard errors for subgroups were often greater than 30\%, the relative standard errors for overall estimates of type 1 diabetes mellitus (T1DM) and type 2 diabetes mellitus (T2DM) were less than $30 \%$, and the relative standard error for T1DM plus T2DM was less than $20 \%$. Concern was also expressed about the low absolute number of cases identified in the study. Accordingly, we did not draw conclusions based on absolute numbers. Nevertheless, presenting the absolute numbers was important (and specifically requested during peer review) to emphasize the need for cautious interpretation of results.

Dabelea et al. (2) comment on the potential for both diabetes overestimation and diabetes type misclassification due to the following factors: 1) participant self-reporting of physician diagnosis was considered sufficient to define a positive case; 2 ) only 1 fasting glucose test result was available for determining undiagnosed disease; and 3) the definition of diabetes type was based solely on medications used by the participant, and no medication or any oral hypoglycemic use was defined as T2DM, whereas only insulin use was defined as T1DM.

Regarding the potential misclassification of diabetes type, we agree that this is a limitation, as outlined in our original article (3). In fact, to comprehensively address the possible magnitude of misclassification, we presented results based on an alternative method for assigning diabetes type as a sensitivity analysis. Specifically, in our sensitivity analysis, any insulin use (with or without use of oral hypoglycemic agents) was defined as T1DM; this alternative method did not meaningfully affect our estimates, which suggests that the potential influence of misclassification was minimal. Moreover, the possibility of misclassification in the Search for Diabetes in Youth Study (herein, the SEARCH Study) also should not be overlooked. Although determination of diabetes type is based on health care provider assignments - a very high standardthe SEARCH Study investigators and others have noted the increasing complexity of assigning diabetes type in light of the obesity epidemic $(4,5)$. 